\title{
Use of Cohesive Elements in Fatigue Analysis
}

\author{
Leonard J. Lucas ${ }^{1}$, T. M. Black, D. P. Jones* \\ ${ }^{1}$ California Institute of Technology \\ Bechtel Bettis, Inc., West Mifflin \\ *Member, ASME
}

\begin{abstract}
Cohesive laws describe the resistance to incipient separation of material surfaces. A cohesive finite element is formulated on the basis of a particular cohesive law. Cohesive elements are placed at the boundary between adjacent standard volume finite elements to model fatigue damage that leads to fracture at the separation of the element boundaries per the cohesive law. In this work, a cohesive model for fatigue crack initiation is taken to be the irreversible loadingunloading hysteresis that represents fatigue damage occuring due to cyclic loads leading to the initiation of small cracks. Various cohesive laws are reviewed and one is selected that incorporates a hysteretic cyclic loading that accounts for energetic dissipative mechanisms. A mathematical representation is developed based on an exponential effective load-separation cohesive relationship. A three-dimensional cohesive element is defined using this compliance relationship integrated at four points on the mid-surface of the area element. Implementation into finite element software is discussed and particular attention is applied to numerical convergence issues as the inflection point between loading and unloading in the cohesive law is encountered. A simple example of a displacementcontrolled fatigue test is presented in a finite element simulation. Comments are made on applications of
\end{abstract}

the method to prediction of fatigue life for engineering structures such as pressure vessels and piping.

\section{Introduction}

This document addresses the implementation of cohesive elements into a finite element package that contain hysteretic mechanisms to ultimately account for void nucleation and fatigue crack initiation due to cyclic loading. The fundamental law in crack growth analyses is Paris' law, a phenomenological law that determines crack growth rates for long cracks under constant loading cycles. Many times the loading history is not so convenient- it may vary in amplitude. Also, analyses may be important near short cracks, or a region of suspected void initiation and/or small crack nucleation. As stated in $[2]^{1}$, ad hoc procedures exist for short cracks and overloading for a specific material and loading combination, but ultimately fail when compared to experimental data for other materials and loadings. Cohesive elements serve to eliminate the need to develop ad-hoc rules governing fatigue analyses by following a cohesive law equipped with damage accumulation and energy dissipation.

The cohesive element presented here is a useful com-

\footnotetext{
${ }^{1}$ Numbers in brackets refer to the list of references.
} 
putational tool for crack and nucleation calculations because of its traction-displacement cohesive law which is independent of geometry and loading history cases. This paper will present a means to perform crack initiation/growth analyses in a finite element setting. Any highly functional finite element package can be used to implement cohesive elements into a finite element framework. We chose to use ABAQUS ${ }^{\circledR}$, which currently offers a cohesive element with different cohesive laws. This report presents a cohesive law that is motived by the hysteretic phenomena that cause decohesion from fatigue cycling. It can be added to any finite element package's suite of cohesive laws.

Reference [1] provides the basis for the cohesive law to be presented here. This report bridges the phenomena that cause fatigue decohesion and the math governing the law, and details the methods attempted to obtain numerical convergence with examples. Further work will yield real applications of the cohesive law to fatigue specimens.

This document is organized as follows. Existing cohesive laws and their relevant features are presented in §3. The mathematics behind the cohesive law are discussed in $\$ 4$. Convergence issues with the cohesive laws are discussed in $\$ 5$. An example problem is presented in $\$ 6$ of a constant amplitude displacementcontrolled fatigue test. Lastly, observations of the current cohesive model and future suggestions are made for improving it in $\S 7$.

\section{Nomenclature and Conven- tions}

The conventions adopted throughout this paper are given in the table below. Note here that tractions and separations in bold font denote effective values as discussed in $\$ 4$, while normal type-set quantities denote single-mode values.

Table 2.1: Operator Conventions

\begin{tabular}{|c|l|l|}
\hline Operator & Name & Representation \\
\hline\langle\rangle & $\begin{array}{l}\text { Macauley } \\
\text { bracket }\end{array}$ & $\langle x\rangle=\left\{\begin{array}{l}x, x \geq 0 \\
0, x<0\end{array}\right.$ \\
\hline$\|\cdot\|_{p}$ & p-norm & $\|\mathbf{x}\|_{p}=\left\{\sum_{i=1}^{2 o r 3} x_{i}^{p}\right\}^{\frac{1}{p}}$ \\
\hline
\end{tabular}

Table 2.2: Symbol Conventions

\begin{tabular}{|c|l|}
\hline Symbol & Value \\
\hline $\mathbf{T}$ & Effective traction \\
\hline $\mathbf{T}_{c}$ & Critical effective traction \\
\hline $\boldsymbol{\Delta}$ & Effective separation \\
\hline $\boldsymbol{\Delta}_{c}$ & Critical effective separation \\
\hline $\boldsymbol{\Delta}_{p}$ or $\boldsymbol{\Delta}_{u}$ & Ultimate effective separation \\
\hline $\mathbf{d}$ & Damage parameter \\
\hline$N$ & Number of fatigue cycles \\
\hline$G_{c}$ & $\begin{array}{l}\text { Critical energy for modal separa- } \\
\text { tion }\end{array}$ \\
\hline$I, I I, I I I$ & Cracking mode \\
\hline$\alpha$ & $\begin{array}{l}\text { Qauntity relating modal proper- } \\
\text { ties to effective properties }\end{array}$ \\
\hline$\beta$ & $\begin{array}{l}\text { Exponential cohesive law sharp- } \\
\text { ness factor }\end{array}$ \\
\hline
\end{tabular}

\section{Cohesive Law}

A cohesive law is a traction-separation curve, similar to an elastic-plastic material law described by a stress-strain curve. The difference in this analogy is that the cohesive law becomes non-monotonic at a critical point, labeled as the critical traction $\left(\mathbf{T}_{c}\right)$ at the critical separation $\left(\boldsymbol{\Delta}_{c}\right)$ point. Here, traction decreases from its maximal value towards zero as separation increases towards catastrophic separation, $\boldsymbol{\Delta}_{u}$ or $\boldsymbol{\Delta}_{p}$. This is often called the softening zone. In this sense, separation is a virtual stretching of a point in a body, and the traction is the force that causes that stretching. When the point separates into two points, a physical void or crack opens in the body and the traction drops to zero accompanied by an indeterminate separation.

Accordingly, a cohesive law is characterized by a monotonically increasing traction-separation curve up to the critical point followed by a monotonically decreasing curve afterwards. Traction decrease is an effect of the material damage due to void generation leading to lessening the capability to resist separation at the interface. This is shown in Figure 3.1 where the material ahead of the crack tip opens but maintains cohesive forces from bridging ligaments. Point $A$ has opened significantly and experiences less traction, while points $B$ and $C$ appear to have opened less and experience more traction. Thus a cohesive law must calculate tractions from separations. Consider a thought experiment to clarify this concept.

Thought Experiment: For clarity of the concept, 


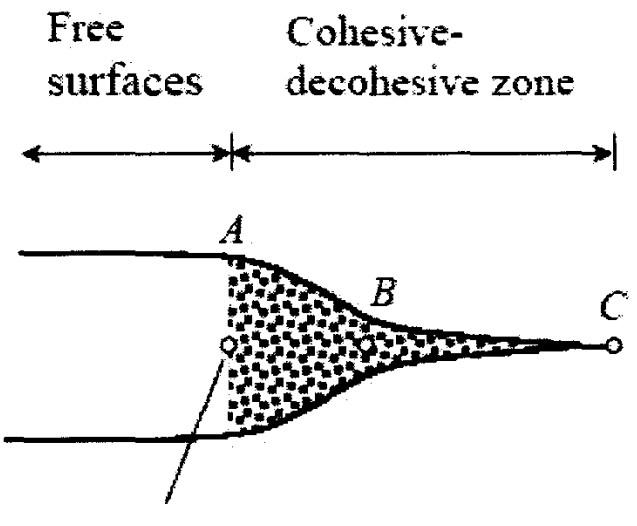

Crack tip

Figure 3.1: Material existing in a "cohesive zone"

imagine a rod being pulled until it fails. If you consider the traction-separation curve, intuition tells you that the rod would maintain some traction as you pull harder, but at some point the material cannot sustain the applied force, and the traction decreases to zero (failure). So the cohesive law must have two distinct features- (zone 1) an elastic-like growth towards a critical separation, and then (zone 2) decreasing traction for increasing separation zone.

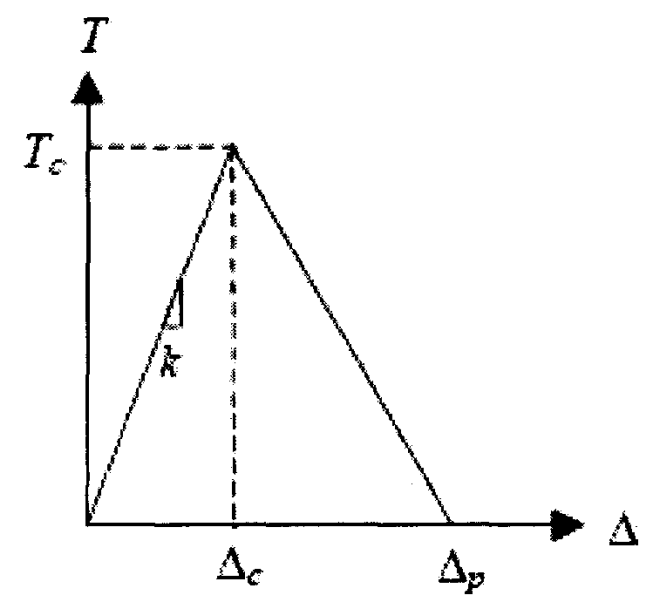

Figure 3.2: The bilinear cohesive law

A few main cohesive laws exist in the literature: a linear law, a bilinear law, (Figure 3.2), an exponential law (Figure 3.3), and a power (polynomial) law (Figure 3.4). The exponential law will be detailed in this presentation- please refer to [1] and [5] for more detailed presentations of the power and linear laws, respectively. Note that the power law has nearly the same shape as the exponential law.

The bilinear law produces solution oscillations near

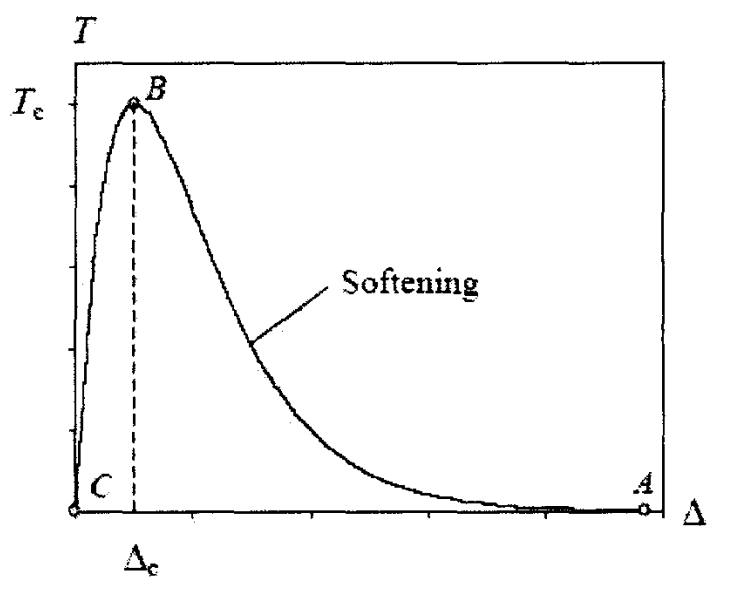

Figure 3.3: Exponential cohesive law in nonnormalized traction vs. displacement

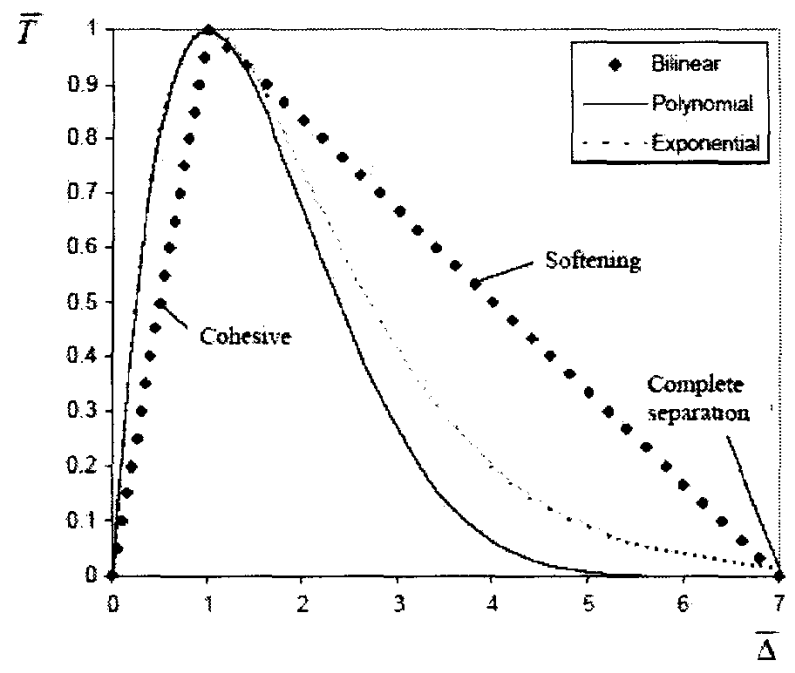

Figure 3.4: Three typical cohesive laws

the transition between zone 1 and zone 2 (cohesive and softening zones, respectively). This happens because the residual force in the Newton-Raphson procedure oscillates near the apex of the bilinear cohesive law. The exponential law is smoother, and thus a more phenomenologically realistic representation of a material behavior towards failure. This is a reason why the linear cohesive law [5] may be preferred over the bilinear law.

The cohesive laws above consider the tractionseparation path for monotonic loading as well as unloading-to-reloading hysteresis. With cyclic loading, one would expect a weakening of the material, as in Figure 3.5. That is, if the specimen is loaded such that the critical separation $\boldsymbol{\Delta}_{c}$ (point 3 ) is surpassed (point 4), and then the material is unloaded (point 
5), the maximum traction would be the traction at maximum separation from the previous loading event (point 6). The material in this case would not fail until the maximum separation, $\boldsymbol{\Delta}_{u}$, is reached and no further force (or traction) is required to produce further separation. Thus the magnitude of the cohesive law degrades, but retains its general shape.

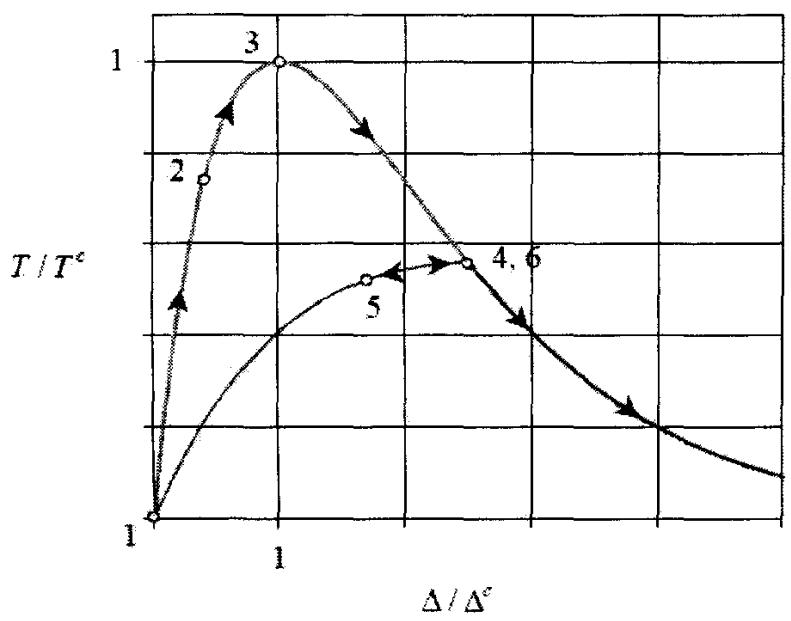

Figure 3.5: Hysteretic reloading paths

A measure of the accumulated degradation of the material is usually referred to as the damage, $d$. Once the material begins to soften, it cannot recover and damage increases monotonically. With this and the previous paragraph, we can deduce:

$$
\mathbf{T}=\mathbf{T}(\boldsymbol{\Delta}, \mathbf{d})
$$

Further, one would expect the material to fail after many loading cycles below the monotonic failure separation limit due to some kind of dissipation. Hysteretic cyclic loadings account for energetic dissipative mechanisms. Reasons are not yet available for these mechanisms based on first principles. However, from a phenomenological point of view, the effect of this on the traction-separation curve is that upon reloading, the maximum traction from the previous cycle cannot be attained, and maximum traction may only reach a slightly lower value for successive reloading events. The number of cycles, $N$, incorporates itself into Eq.(3.1) through the following:

$$
\mathbf{T}=\mathbf{T}(\boldsymbol{\Delta}, \mathbf{d}, N)
$$

Effects from $N$ in Eq.(3.2) can also be accounted for by controlling $\mathbf{d}$ upon reloading cycles. As discussed in $\S 4.4$, by controlling $\mathbf{d}$ such that it increases slightly upon reloading, the reloading path travels below the previous unloading path and results in energy dissipation and decrease in maximum sustainable traction (Figure 3.6 and Figure 3.7). The physical phenomenon behind this dissipation and damage degradation cannot be modeled in this framework as it is speculated to happen at a smaller scale. Such mechanisms may include dissipation through ligaments bridging material in the cohesive zone and/or crystallographic slip, as suggested in [2].

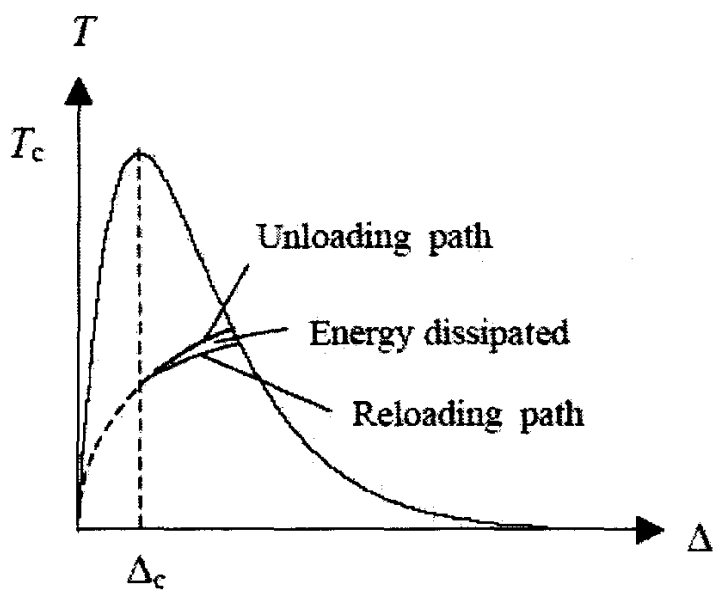

Figure 3.6: One cycle demonstrating energy dissipating mechanisms

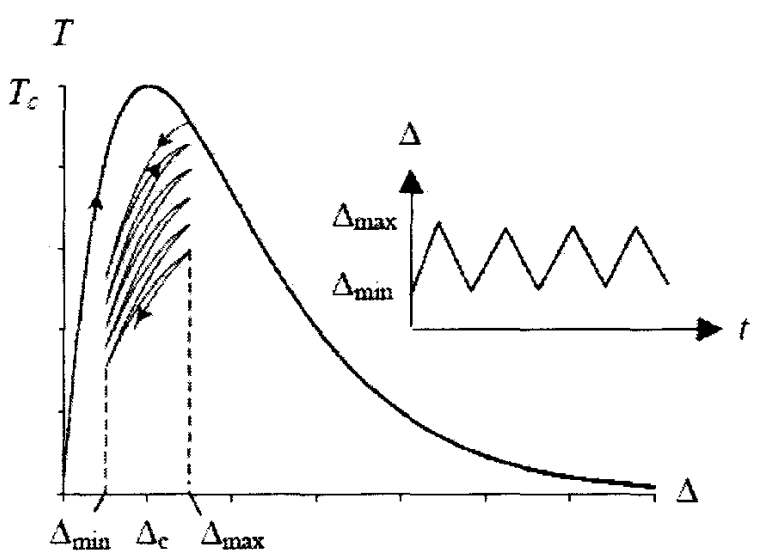

Figure 3.7: Multiple cycles demonstrating energy dissipating mechanisms

Separation is calculated at the integration points of the cohesive element per conventional element calculations. The integration points conventionally lie in the midplane of the cohesive element, as in Figure 3.8. 


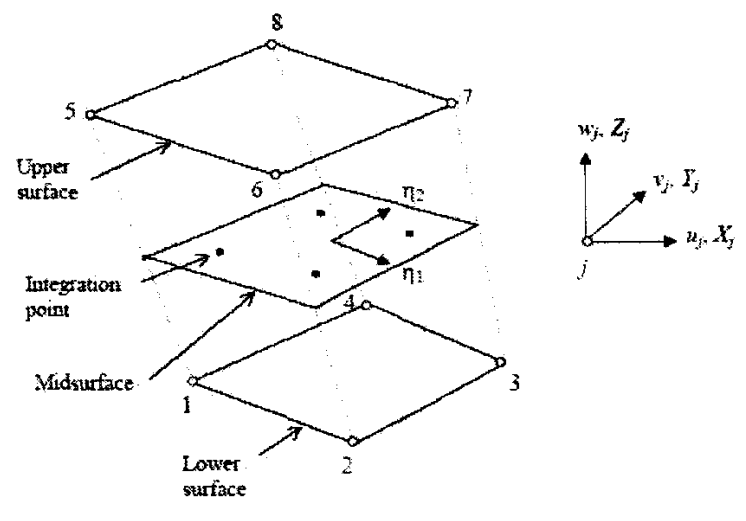

Figure 3.8: Integration points along midsurface of a cohesive element

\section{Formulation of Element}

The formulation for a cohesive element begins with the notion of an effective traction versus effective separation curve. There are a few ways to define the independent variable, effective traction. The most common is based on the different modes of crack formation. Consider the crack initiation model to apply to an inclusion in the material. Then expanding the dimension of the inclusion can be thought of analogously to the opening of a crack. As in fracture mechanics, the opening mode, Mode $I$, can play both a tensile and compressive role in the law, while the sliding and tearing modes, Mode $I I$ and Mode III respectively, are not subjected to the tensile or compressive description. Thus we seek a law that accounts for primarily Mode I tensile failure behavior. Since the cohesive model applies to a virtual separation of a point we can also think of a Mode $I$ compressive separation.

In one-dimensional analysis, effective separation is simply separation in the single direction. In higher physical dimensions the effective separation can be computed through a norm, (e.g. a p-norm, $\|\cdot\|_{p}$ ) that would give an effective length in an orthonormal basis. For each mode, we will need to define the cohesive law based on its fracture parameters, then relate these independent modal laws to one effective law. We begin by identifying the properties needed to define the modal exponential cohesive laws in $\$ 4.1$, and then combining these into an effective cohesive law in $\S 4.2$.

\subsection{Cohesive Law Properties}

Three properties exist (two of which are independent) that describe the cohesive law for each failure mode.
These are the critical values for the energy of separation, effective traction, and effective separation $\left(G_{c}\right.$, $T_{c}, \boldsymbol{\Delta}_{c}$ respectively). Total separation energy is defined as the area under the traction-separation curve, and is the energy needed to generate a void at the point under consideration. $\$ 4.2$ will show how these modal cohesive laws form an effective cohesive law.

Consider Figure 3.6. Traction must have a maximum value, $T_{c}$, at which point the separation has a critical point, $\Delta_{c}$. Note that the first loading cycle where the separation $\Delta$ goes beyond $\Delta_{c}$, each successive cycle will have an updated critical point where the reloading cycle meets the softening regime of the cohesive envelope, obviously depending on how far $\Delta$ has surpassed $\Delta_{c}$. It is at this point in a cycle where a maximum traction is reached, which is equal to or less than the previous cycle's maximum traction. This relegates $\Delta_{c}$ and $T_{c}$ to be parameters that are meant to define the cohesive law envelope, and not critical values for each cycle.

In a bilinear law, given any two of the three critical values described above, the third can be found since the area under the curve corresponds to the area of a triangle Eq.(4.1). In an exponential law, the same is true except that a factor $\psi(\beta)$, Eq. (4.2), is needed to calibrate the area to give a triangular-like relationship Eq. (4.3) between the variables in the list above. Here, $\beta$ is the sharpness factor, and defines the broadness of the exponential law (see Figure 4.1). Increasing $\beta$ corresponds to a "tighter" exponential law, while decreasing $\beta$ corresponds to a "fatter" law.

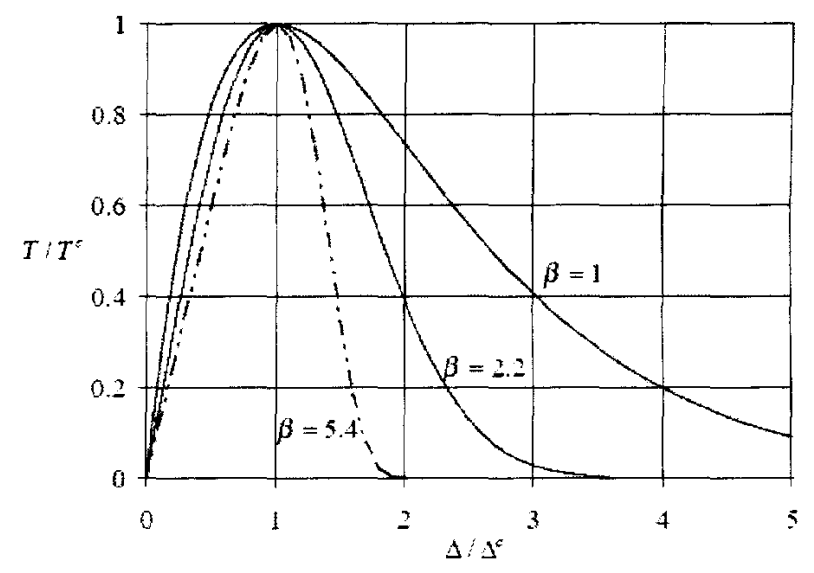

Figure 4.1: Effect of $\beta$ on the exponential cohesive law

$$
G_{c}=\frac{1}{2} T_{c} \Delta_{c}
$$




$$
\begin{gathered}
\psi(\beta)=\exp \frac{1}{\beta} \Gamma\left(\frac{2}{\beta}\right) \beta^{\frac{2}{\beta}-1} \\
G_{c}=\psi(\beta) T_{c} \Delta_{c}
\end{gathered}
$$

In Eq.(4.2), $\Gamma$ is the $\Gamma$-function with argument $2 / \beta$. Note that $\lim _{\beta \rightarrow \infty} \psi(\beta)=1 / 2$. Thus larger values of $\beta$ assimilate a bilinear cohesive law with $\Delta_{c} \rightarrow \Delta_{p}$. This limit corresponds to a material with perfect brittleness, or no plasticity mechanism. Now one can obtain the shape of each modal cohesive law based on two of three fracture properties. Having multiple active modes leads us to develop a cohesive law that combines the effects of all the possible active modes that will experience separation. The next section defines an effective cohesive law for effective traction versus effective separation.

\subsection{Effective Separation}

Consider we are at some state in the finite element simulation, and there is non-zero separation for each mode at some point. Define an effective separation as follows:

$$
\begin{aligned}
e_{e f} \bar{\Delta} & =\left(\left\langle\bar{\Delta}_{I}\right\rangle^{\alpha}+\left|\bar{\Delta}_{I I}\right|^{\alpha}+\left|\bar{\Delta}_{I I I}\right|^{\alpha}\right)^{\frac{\beta}{\alpha}} \\
\Delta & \equiv e f f \bar{\Delta}
\end{aligned}
$$

Some comment is warranted regarding the meaning of Eq.(4.4). For shorthand we use Eq.(4.5) to define any normalized effective separations- those left un-bolded represent modal separations. Inside the square brackets, we have an expression similar to an $\alpha$-norm for each $\bar{\Delta}_{i}$ where $i=\{I, I I, I I I\} . \bar{\Delta}_{i}$ is the normalized separation for the $i$-th mode, or $\Delta_{i} / \Delta_{i C}$. Note this value can take values in $(-\infty, \infty)$ for modes $I I$ and $I I I$, but the designation with the Macauley brackets for mode $I$ indicates only positive values, $(0, \infty)$. Thus compressive mode $I$ does not contribute to the fracturing of the material. The power outside of the square brackets indicates a dependence on $\beta$ and the power $\alpha$. Thus for $\alpha=2$, this is the Euclidean norm to the $\beta$ power.

Note that $\boldsymbol{\Delta}$ is the normalized effective separation. The significance of using $\bar{\Delta}_{i}$ instead of $\Delta_{i}$ in Eq. (4.4) is that we don't need to specify $e f f \Delta_{C}$. That is, working in the normalized separation space (Eq.(4.4)) is more convenient than Eq.(4.6).

$$
\Delta \equiv e_{e f} \bar{\Delta}=\frac{\left[\left|\Delta_{I}\right|^{\alpha}+\left|\Delta_{I I}\right|^{\alpha}+\left\langle\Delta_{I I I}\right\rangle^{\alpha}\right]^{\frac{\beta}{\alpha}}}{e f f \Delta_{C}}
$$

\subsection{Damage Effects on the Cohesive Law}

With $\boldsymbol{\Delta}$, we can account for mode mixity effects and follow a distinct traction-separation curve. Of course this mode mixity is unimportant in single mode opening, and $\boldsymbol{\Delta}$ reflects this by reducing to the active modal cohesive law. Now consider from arguments in $\S 3$ that cohesive laws depend on a damage parameter. This monotonically increasing parameter is a measure of accumulated irreversible cohesive effects. It can be formulated as:

$$
{ }^{t+\delta t} \mathbf{d}=\max \left(1,{ }^{t} \mathbf{d},{ }^{t+\delta t} \boldsymbol{\Delta}\right), \quad{ }^{0} \mathbf{d}=1
$$

This equation states that the updated damage is the maximum $\Delta$ attained up to the current time step. Upon unloading, $\mathbf{d}$ retains the value of the previous maximum $\boldsymbol{\Delta}$ accumulated. No damage accumulates until the critical separation is reached $\left(\right.$ eff $\bar{\Delta}={ }_{\text {eff }} \bar{\Delta}_{c}$ or $\Delta=1$ ).

One expects to travel along the monotonic cohesive envelope before the softening zone (zone 2) is ever attained, and upon reloading past the previous cycle's maximum effective separation. The cohesive law should mathematically allow for travel along the monotonic envelope in the cases of $\mathbf{d}=1$ and $\Delta=\mathbf{d}$. The expression $(2-\boldsymbol{\Delta} / \mathbf{d}-\mathbf{d})$ gives this property. Both $\mathbf{d}=1$ and $\boldsymbol{\Delta}=\mathbf{d}$ give $(1-\mathbf{d})$ when Eq. $(4.7)$ is considered.

\subsection{Hysteretic Cyclic Dissipation}

Hysteretic energetic dissipative mechanisms drive fatigue failure, and can be included in the exponential cohesive law model ${ }^{2}$. This can be done by slightly increasing the damage parameter upon reloading. By introducing the parameters $n$ and $\lambda$ associated with fatigue crack growth data, the following expressions can equip the cohesive law with a dissipative mechanism to achieve fatigue crack grow $\mathrm{h}^{3}$ :

Loading

$$
{ }^{t+\delta t} \mathbf{d}=\max \left(1,{ }^{t} \mathbf{d},{ }^{t+\delta t} \boldsymbol{\Delta}\right)
$$

\footnotetext{
${ }^{2}$ Please refer to [5] for a complete formulation and calculation of these effects on the linear cohesive law.

${ }^{3}$ Note that $[1]$ alters this expression by using $\Delta_{\max }$ instead of $\boldsymbol{\Delta}_{\min }$ and swtiching the subtractive expressions that are raised to the $n$ power in order to achieve dissipative effects on the unloading half of the cycle. The expression given in Eq. (4.9) reflects dissipation for the loading half of the cycle.
} 


$$
\begin{aligned}
& \text { IF }\left(\left({ }^{t+\delta t} \mathbf{d}>1.0\right) \text {.AND. }\left({ }^{t+\delta t} \boldsymbol{\Delta}<{ }^{t+\delta t} \mathbf{d}\right)\right) \text { THEN } \\
& { }^{t+\delta t} \mathbf{d}={ }^{t+\delta t} \mathbf{d}+\lambda\left(\left({ }^{t+\delta t} \boldsymbol{\Delta}-\boldsymbol{\Delta}_{m i n}\right)^{n}-\left({ }^{t} \boldsymbol{\Delta}-\boldsymbol{\Delta}_{m i n}\right)^{n}\right)
\end{aligned}
$$

$\underline{\text { Unloading }}$

$$
\boldsymbol{\Delta}_{\min }={ }^{t+\delta t} \boldsymbol{\Delta}
$$

For a given loading between some $\boldsymbol{\Delta}_{\min }$ and $\boldsymbol{\Delta}_{\max }$ with $\lambda \neq 0$, the law behaves like that pictured in Figure 3.7. Note that the loading is not constrained between $\boldsymbol{\Delta}_{\min }$ and $\boldsymbol{\Delta}_{\max }$ and the fatigue effects in the law can account for overloading and underloading without applying ad-hoc rules outside the predictive capability of Paris' Law. Further inspection shows for constant load range cycles, $\boldsymbol{\Delta}_{\min }<\boldsymbol{\Delta}<\boldsymbol{\Delta}_{\max }$, the damage growth rate resembles that of crack growth under Paris' Law:

$$
\frac{\partial \mathbf{d}}{\partial N} \approx \frac{\mathbf{d}_{N+1}-\mathbf{d}_{N}}{(N+1)-N}=\lambda\left(\Delta_{\max }-\Delta_{\min }\right)^{n}
$$

The parameters $\lambda$ and $n$ must be chosen to match the test data of a certain material or specimen. Further research is needed to bridge atomic scale phenomena to macro-scale phenomena that govern crack growth both inside and outside of the Paris Law regime. Typical values of $\lambda$ will be very small $\left(10^{-5}<\lambda<10^{-8}\right)$. This range is not strict, but should give the user some intuition about the value of $\lambda$.

The parameter $n$ suggests a relationship similar to the slope in the $\log$ - $\log$ Paris crack-growth correlation. Paris' Law is dependent upon knowing the applied stress intensity factor range to calculate the crack growth rate, and thus the cohesive law appears ill-equipped to relate to Paris' Law. In particular, the Paris Law is fundamentally related to linear elastic material beavior. The following argues a connection between cohesive law calculations and Paris Law calculations.

Consider that the stress intensity factor, $K$, is directly related to the applied load, and then local strain is approximated by the finite-element program through the governing equations. The cohesive law accepts the local strain to calculate the effective separation, $\boldsymbol{\Delta}$. From $\boldsymbol{\Delta}$, damage $\mathbf{d}$ is calculated, which basically tracks the maximum $\boldsymbol{\Delta}$ achieved. With a cyclic loading schedule, $\boldsymbol{\Delta}$ may be within the range $\left[\boldsymbol{\Delta}_{\min }, \boldsymbol{\Delta}_{\max }\right]$, but $\mathbf{d}$ grows with each cycle because of hysteretic dissipation, as per Eq.(4.9). Thus the

\footnotetext{
${ }^{4}$ IF-THEN logical statement in Fortran 77 syntax.
}

change in $\mathbf{d}$ per cycle is given by Eq.(4.11), a monotonic increase. Continual opening of the cohesive element through the evolution of $\mathbf{d}$ eventually leads to element failure and thus crack extension. Thus, $\mathbf{d}$ can be thought of as a measure that tracks the length of the crack, $a$. Taking the $\log$ of both sides of Eq.(4.11) resembles Paris' Law for $\Delta K=K_{\max }-K_{\min }$ related to $\Delta \boldsymbol{\Delta}=\boldsymbol{\Delta}_{\max }-\boldsymbol{\Delta}_{\min }$ and $\partial a / \partial N$ related to $\partial \mathrm{d} / \partial N$.

Comparing the crack growth rate to Paris' Law for a valid crack regime in a finite element analysis may be a valuable verification method for cohesive elements.

\subsection{Complete Exponential Cohesive Law}

The previous subsections are presented to provide meaning to the complete exponential cohesive law expression. In the implicit iterative Newthon-Raphson procedure, the state of the material must be updated. Therefore, the tractions at the integration points of the cohesive elements must be computed, and the updated tangent stiffness matrix must be given back to the Newton-Raphson scheme to iterate to the next state. The tractions in the modal directions are given by Eq.(4.12):

$$
T_{i}=T_{i_{c}} \bar{\Delta}_{i} \exp (2-\mathbf{\Delta} / \mathbf{d}-\mathbf{d}), \quad i=\{I, I I, I I I\}
$$

The effective cohesive law is obtained from this equation. By replacing the modal terms with effective terms, the effective cohesive law becomes:

$$
\mathbf{T}=\boldsymbol{\Delta} \exp (2-\boldsymbol{\Delta} / \mathbf{d}-\mathbf{d})
$$

Taking the derivative of Eq. (4.13) with respect to each of the modal separations gives the tangent stiffness matrix (see [1] for more details). The form of the tangent stiffness matrix, or Jacobian, is given by Eq.(4.5):

$$
\begin{gathered}
\frac{\partial T_{i}}{\partial \Delta_{j}}=\left(\delta_{i j}-\frac{\bar{\Delta}_{i} \bar{\Delta}_{j}\left|\bar{\Delta}_{j}\right|^{\alpha-2} \mathbf{\Delta}^{(1-\alpha / \beta)}}{\tilde{w}}\right) \times \\
\exp (2-\boldsymbol{\Delta} / \mathbf{d}-\mathbf{d}) \\
\text { where } \quad i, j=\{I, I I, I I I\} \\
\tilde{w}= \begin{cases}1, & \mathbf{d}<\boldsymbol{\Delta} \\
\mathbf{d}, & \mathbf{d} \geq \boldsymbol{\Delta}\end{cases}
\end{gathered}
$$


Here, $\delta_{i j}$ is the Kroenecker delta $\left(\delta_{i j}=1\right.$ if $i=j$, 0 otherwise). The tractions and tangent stiffness matrix are consistent with the hysteretic dissipation, reloading hysteresis, and monotonic envelope loading since all of that information is updated and contained within the parameters $\boldsymbol{\Delta}$ and $\mathbf{d}$.

In the case of compression in Mode $I\left(\bar{\Delta}_{I}<0\right)$, the cohesive element applies a stiffness that never experiences a softening. This is the case because compression will not cause a material interface to decohere unless buckling is accounted for (it is not in this model). Otherwise, compression does not increase $\mathbf{d}$ in this model. It may be argued that compressive loadings may cause rewelding of the material. If the rewelding is significant, one can incorporate a damage relaxation once $\Delta$ is in the compressive region.

With this, the implementation of the cohesive law is complete, but a numerical convergence problem exists at the apex into the softening regime. This issue and some techniques to overcome this are presented in $\$ 5$.

\section{Comments on Implementa- tion}

The convergence properties of a cohesive model depend significantly on the type of loading. Without viscous stabilizing parameters, pressure loadings cannot advance past the peak of the cohesive law, while displacement loadings result in a well-defined problem, leading to convergence past the peak.

Both the bilinear and exponential cohesive laws have convergence issues at the critical effective separation. As discussed in [1], the bilinear law produces significant solution oscillations at the discontinuity. This is the primary reason for using the exponential law as it provides the smoothness required to subside these oscillations. Unfortunately, the exponential law reaches a zero material tangential stiffness matrix at the peak, and numerical techniques must be employed to allow the solution to cross this barrier when using pressure loads. This is the case for any material exhibiting nonlinearities, see Figure 5.1 for an extreme example of this. Cohesive laws have softening regimes that causes numerical problems in an implicit iterative scheme.

Pressure loadings are ill-posed problems for softening laws such as the cohesive law. This is the case because when the pressure load overcomes the critical traction $\mathbf{T}_{c}$, the tangent stiffness is essentially zero. Thus, the Newton-Raphson method (see Figure 5.2) is not able to converge upon a subsequent set of separations

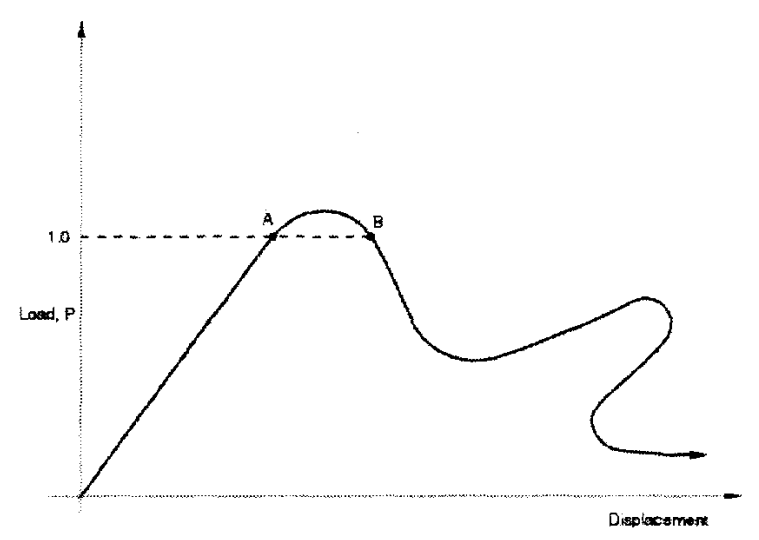

Figure 5.1: A nonlinear material law showing difficult convergence regimes

that satisfy the condition for a low force residual in the analysis. The analysis converges very slowly to the peak of the cohesive law and cannot continue to define a separation that gives a low force residual (see the analysis abortion at the peak of the cohesive law in Figure 5.4). This happens because the tangent stiffness cannot advance from zero values to negative values in an iterative scheme. It is easy to observe the suggested separation increment blows up near the peak as the tangent stiffness suggests a zero derivative that any set separations satisfies the update but does not produce a low residual.

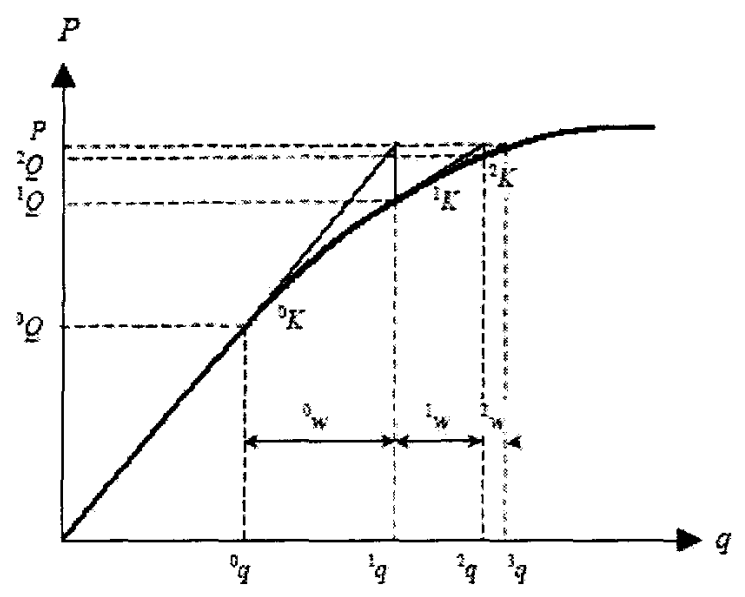

Figure 5.2: Successive iterations for an increment in a Newton-Raphson scheme

The implementation that [1] uses attempts to overcome pressure-loaded convergence problems by using different stiffnesses (see Figure 5.3). The tangent stiffness gives the numerical problem described above. One can also use a secant stiffness or a pseudo stiffness. The secant method is a line from the origin to 
the current state of the material. With very small iterations, the secant stiffness may converge, but the residual tolerances and the incrementing schedule may have to be changed against the user's will. According to [1], the pseudo stiffness works best near the critical point, with variations that give different paces of convergence. The pseudo stiffness is made by inserting zeroes in the stiffness matrix for any term involving the normal mode except the pure mode $I$ derivative. Zeroes can be placed in the stiffness matrix at the local coordinate level or at the global coordinate level. This method provides numerical difficulties and did not result in converged solutions.

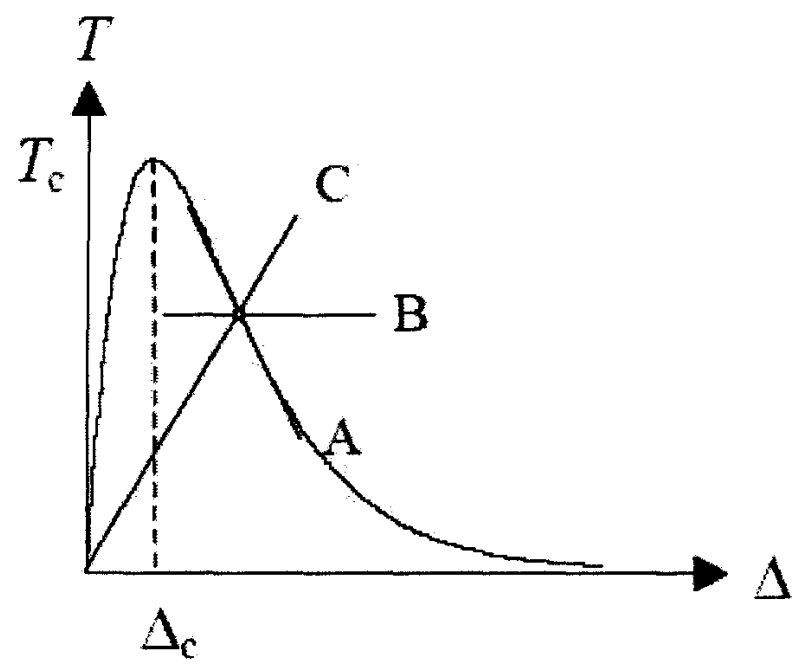

Figure 5.3: Three stiffnesses used for solution convergence: Tangent stiffness (A), Pseudo stiffness (B), Secant stiffness (C)

In highly functional FE packages, options exist to obtain a converged solution using pressure loadings. As a specific example in ABAQUS ${ }^{\circledR}$, any step that reaches/passes the cohesive law peak (and all subsequent steps), one can employ the STABILIZE option. This parameter introduces automated viscous forces that are sufficiently large to prevent instantaneous instability but small enough not to significantly affect the behavior when the problem is stable, or the user can define it uniquely. Figure 5.5 below shows the effect of viscous damping on a test specimen. This figure shows the first loading cycle $(0<$ Time $<1)$ nearing the critical point of the cohesive law, and then a subsequent reloading $(2<$ Time $<3)$ past the critical point, and a third reloading $(4<$ Time $<5)$ that is the same as the second loading. All unloadings are set to a pressure of magnitude zero. Without hysteresis, we should expect that the second and third loadings produce the same results, but this is not the case because the stabilizing parameter intro- duces viscous forces that distort the solution significantly. Also note that $\boldsymbol{\Delta}$ and $\mathbf{T}$ lag behind their expected trajectories for the given loadings. The second reloading reaches its peak at Time $\approx 3.5$, not at 3.0. The same behavior happens for the unloading half of the cycles since the stabilizing parameter is used to retreat back over the apex. Because of the solution distortion and lag, the stabilizing parameter is not an acceptable method to control convergence of the cohesive element beyond the critical point for pressure loadings.

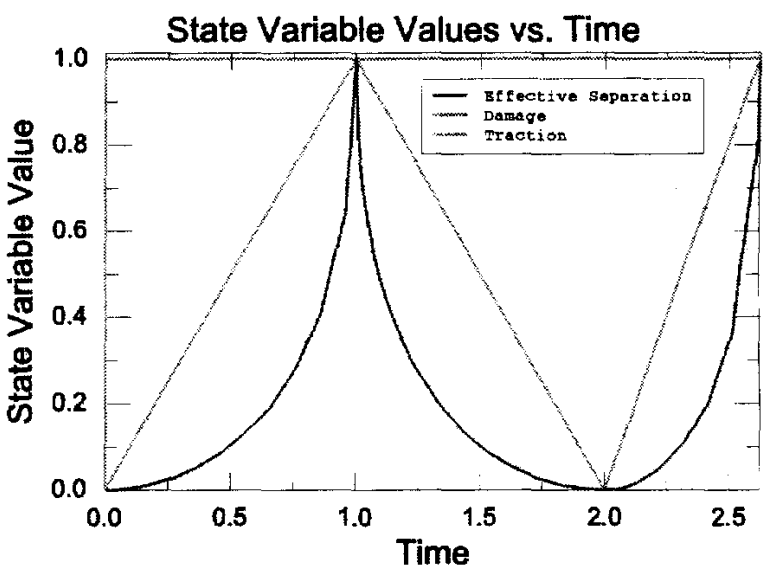

Figure 5.4: Results showing abortion of a pressureloaded cohesive element analysis because of nonconvergence

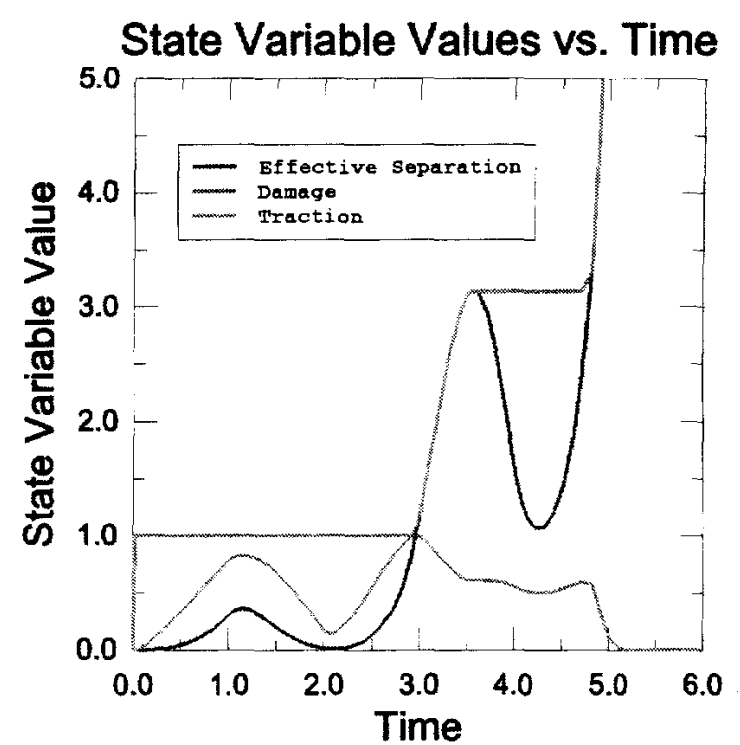

Figure 5.5: The effect of the stabilizing parameter on a pressure-loaded specimen

Viscous regularization of the damage parameter can be employed. The viscous damage evolves in each 
loading stage according to the differential equation defined in Eq.(5.1a), which leads to Eq.(5.1b) for the conditions on the exponential law:

$$
\begin{gathered}
\dot{\mathbf{d}}_{v i s c}=\frac{1}{v}\left(\mathbf{d}-\mathbf{d}_{v i s c}\right) \\
\mathbf{d}_{v i s c}=\max (1, \mathbf{d}(1-\exp (-t / v))
\end{gathered}
$$

Here, $t$ is the fraction of time completed for the time step (which holds no physical meaning in a static analysis except that it increases during a step). At the beginning of the step, $\mathbf{d}_{v i s c}$ starts as 1 and evolves to its expected value $\mathbf{d}$ as $t \rightarrow \infty$. By using $\mathbf{d}_{v i s c}$ instead of $\mathbf{d}$ in the expressions for tractions, stresses are permitted to be outside the limits defined by the traction-separation cohesive law, allegedly relieving some convergence difficulties. This damage evolution can be implemented in a user-defined material (UMAT in ABAQUS ${ }^{\circledR}$ ), but has been turned off for the displacement loadings since convergence is satisfactory without using Eq. (5.1). Typical values of $v$ are $\sim 10^{-3}$, lower values decrease the disparity between $\mathbf{d}$ and $\mathbf{d}_{v i s c}$. Experience shows that pressure loaded specimens show no better convergence results with viscous regularization.

Displacement loadings prescribe the incremented separation that allows convergence in the softening regime, with much faster convergence near the peak of the cohesive law since the iterated separations are informed from the loading and maintain a low force residual. Displacement loading is used in the example in the next section.

\section{Application to Displacement Controlled Fatigue Test}

The examples presented here show a displacementloaded bar with a through-all layer of cohesive elements (see Figure 6.1). They are oriented such that they experience mode $I$ opening when the bar is displaced along the length of the bar.

The cohesive material properties, non-cohesive material properties, and analysis parameters used in the model are given below in Tables 6.1-6.3.:

The parameters $\alpha, \beta, \Gamma, n$ are chosen according to the choices made by [1]. This example uses a large value of $\lambda$ to display the effects of hysteretic energy dissipation for few cycles. $E, \nu$, and $\sigma_{y}$ are the elastic modulus, Poisson ratio, and yield stress of the perfectly plastic non-cohesive stainless steel hexahedral elements. The parameter $v$ is the viscous regularization parameter described in $\$ 5$. The choices for

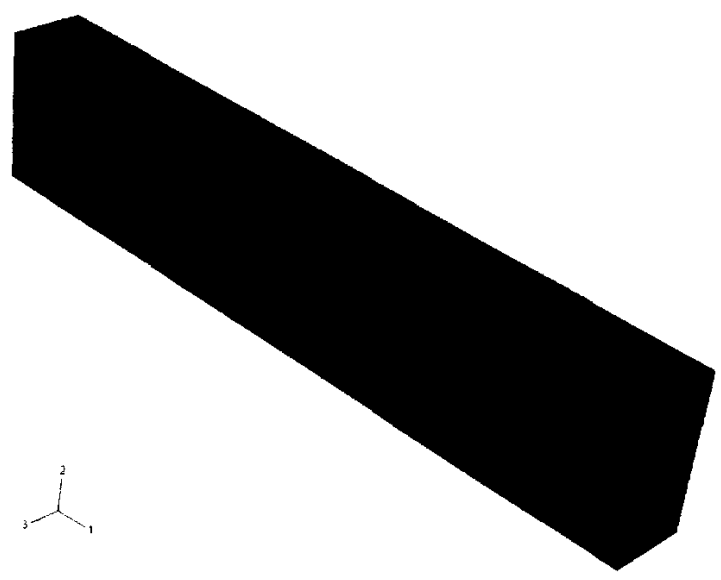

Figure 6.1: Bar used for example analysis (cohesive elements highlighted in center)

Table 6.1: Cohesive Properties used for example

\begin{tabular}{|l|l|}
\hline Parameter & Value \\
\hline$G_{c}(I)$ & 0.35 (in-lbf) \\
$G_{c}(I I)$ & 1.45 (in-lbf) \\
$G_{c}(I I I)$ & 1.45 (in-lbf) \\
$T_{c}(I)$ & 50.0 (psi) \\
$T_{c}(I I)$ & 60.0 (psi) \\
$T_{c}(I I I)$ & 60.0 (psi) \\
$\alpha$ & 2.0 \\
$\beta$ & 2.0 \\
$\Gamma[2 / \beta]$ & 1.0 \\
$\lambda$ & 0.06 \\
$n$ & 2.0 \\
$\Delta_{u}$ & 12.0 \\
\hline
\end{tabular}

modal values of $G_{c}$ and $T_{c}$ are taken from soft cohesive laminate material properties in [1] and do not represent the metallic properties of the surrounding non-cohesive material. The experiment represents two stainless-steel bars "glued" together with a glue having modal properties $G_{c}$ and $T_{c}$.

The setup and results of this analysis are designed to show basic functionality of the cohesive elements for displacement loadings. The contiguous material is much stronger than the cohesive material, giving an essentially rigid response. The cyclic loadings and their discussion is presented in Table 6.4:

As a check for the whole model behaving appropriately, Figure 6.4 shows the reaction force in the displaced direction at the clamped end (center node of that face). We should expect that the reaction force follows the traction of the cohesive element propor- 
Table 6.2: Non-cohesive properties used for example

\begin{tabular}{|l|l|}
\hline Parameter & Value \\
\hline$E$ & $2.83 \mathrm{e}+07(\mathrm{psi})$ \\
$\sigma_{y}$ & $28179.0(\mathrm{psi})$ \\
$\nu$ & 0.3 \\
\hline
\end{tabular}

Table 6.3: Numerical analysis parameters

\begin{tabular}{|l|l|}
\hline Parameter & Value \\
\hline$v$ & $1.0 \mathrm{e}-10$ \\
\hline
\end{tabular}

tionally since the internal forces must be balanced and the contiguous material is essentially rigid. A visual inspection shows that this is the case. Therefore this model validates the use of cohesive elements for displacement-loaded Mode $I$ analyses Further tests can be done with mixed-mode analyses, and cohesive mesh sensitivities in the active regions (see $\$ 7$ below for suggestions on further work and applications.

\section{Discussions on Applications to Pressure Vessel Design}

It is the hope to insert these versatile cohesive elements into a fatigue analysis and accurately reproduce test data. The intended application is for fatigue life predictions of existing pressure vessel components and improving the fatigue life of new pressure vessel designs. The idea is to tune the material parameters from one set of data and then use those parameters to predict the life of another geometry and loading configuration. With such a capability, it will be possible to numerically investigate the fatigue strength a component rather than doing an expensive experimental program. It is also intended to be used for modeling void formation and coalescence for crack initiation studies, as well as analyses for short crack growth. The model may also be used to investigate the life of a specimen after a long crack has developed. As in Figure 7.1, the life of a specimen may last a significantly long time after crack detection.

\subsection{Discussion}

Research on cohesive elements is becoming quite common in academia, and much is becoming known about their convergence behavior in an iterative scheme. Convergence also depends on the mesh size of the cohesive elements that may be experiencing softening.
Table 6.4: Description of loading cycles used to obtain results in Figure 6.3 and Figure 6.2

\begin{tabular}{|c|c|c|}
\hline Cycle & Time & Description \\
\hline 1 & {$[0,2)$} & $\begin{array}{l}\text { Loading below the critical } \\
\text { value, unloading to zero. Note } \\
\text { that the loading remains on } \\
\text { the monotonic envelope, so } \\
\text { remains uninitiated. }\end{array}$ \\
\hline 2 & {$[2,4)$} & $\begin{array}{l}\text { Reloading past the critical } \\
\text { point }\left(\Delta>\Delta_{c}\right) \text {, unloading } \\
\text { to zero. Figure } 6.3 \text { shows } \\
\text { that } \Delta \text { follows the displace- } \\
\text { ment loading, but that d con- } \\
\text { tinually increases during load- } \\
\text { ing until the unloading starts } \\
\text { because of hysteretic dissipa- } \\
\text { tion. I reflects the in- } \\
\text { crease by unloading along a } \\
\text { lower energy path. Note here } \\
\text { the new critical point is at a } \\
\text { lower traction and higher sep- } \\
\text { aration than cycle } 1 . \text { This } \\
\text { happens when } \Delta_{N}>\Delta_{N-1} \\
\text { where } \Delta_{N} \text { is the largest } \Delta \\
\text { reaches during cycle } N \text {. }\end{array}$ \\
\hline 3 & {$[4,6)$} & $\begin{array}{l}\text { Same loading as the previous } \\
\text { cycle. Note that the continual } \\
\text { increase in lowers the maxi- } \\
\text { mum value. }\end{array}$ \\
\hline 4 & {$[6,8)$} & $\begin{array}{l}\text { Same reloading as the previ- } \\
\text { ous cycle, higher unloading. } \\
\text { Note that } T \text { returns toward } \\
\text { the origin until the non-zero } \\
\text { unloading value is reached. }\end{array}$ \\
\hline 5 & {$[8,10)$} & $\begin{array}{l}\text { Increased reloading, unload- } \\
\text { ing to zero. Note how the } \\
\text { increased loading eventually } \\
\text { forces the } T \text { back onto the } \\
\text { monotonic envelope. }\end{array}$ \\
\hline 6 & {$[10,12)$} & $\begin{array}{l}\text { Same reloading as the previ- } \\
\text { ous cycle but lower unloading. } \\
\text { Evolution of } \mathrm{I} \text { shows contin- } \\
\text { ual degradation following in- } \\
\text { creased } \mathrm{a}\end{array}$ \\
\hline 7 & {$[12,14)$} & $\begin{array}{l}\text { Same reloading as the previ- } \\
\text { ous cycle, unloading to zero. } \\
\text { Evolution of } T \text { shows contin- } \\
\text { ual degradation following in- } \\
\text { creased } 1 \text {. Loading past } \Delta= \\
12.0 \text { will result in decohesion } \\
\text { at this point since } \Delta_{u}=12.0 \text {. }\end{array}$ \\
\hline
\end{tabular}




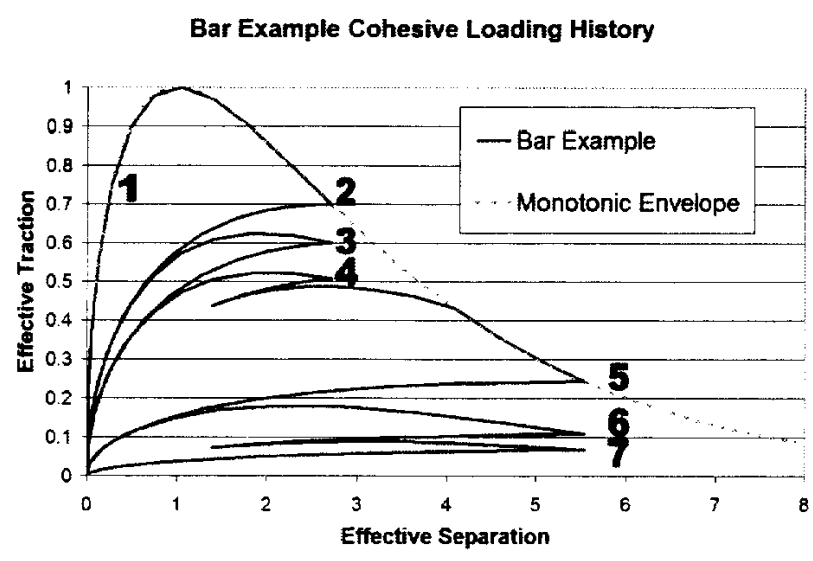

Figure 6.2: Cohesive trajectory of bar example, labeled by cycle at the transition from loading to unloading

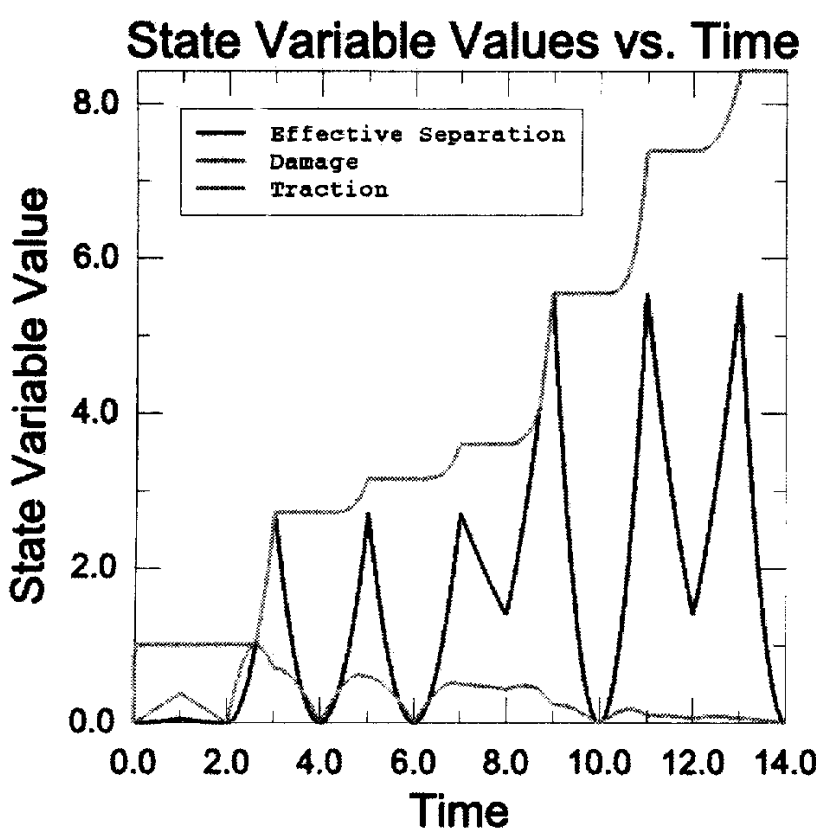

Figure 6.3: State variable results from displacement controlled example

The large stress gradients ahead of the void/crack tip pose a numerical stability problem for coarse meshing. Like in [1], researchers agree that at least two cohesive elements must be present in the active region ahead of the void/crack tip to resolve the stress gradients and produce a smooth solution, otherwise the solution oscillates. The same effect happens if more integration points are chosen over having more cohesive elements in the cohesive region (11]). It is advantageous to have more cohesive elements in the

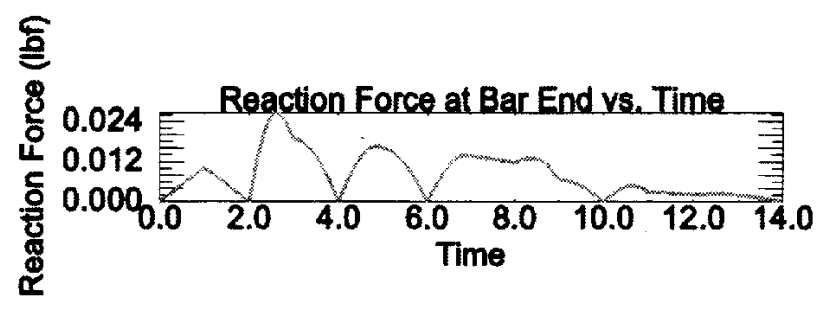

Figure 6.4: Reaction forces at a clamped-end node, compare to "Traction" in Figure 6.3

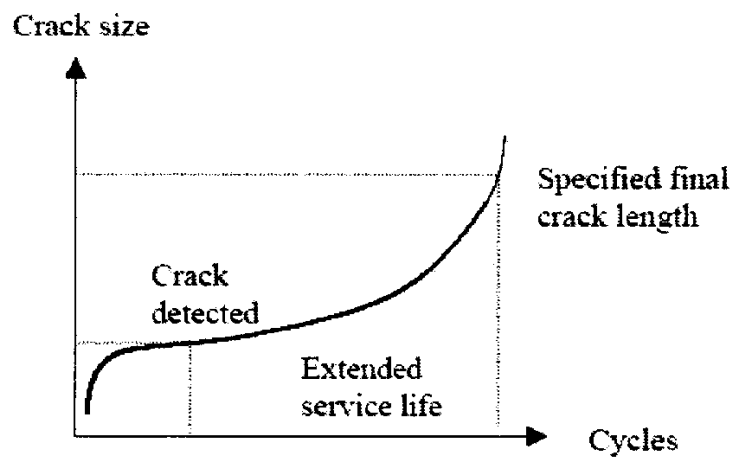

Figure 7.1: Fatigue life of a specimen

cohesive zone than to just increase the number of integration points. At least two fully integrated cohesive elements stifles oscillatory solutions in the decohesion region ([1]).

For crack propagation studies, one may want to produce a tortuous crack path that realistically represents an actual crack seen in experiments. It is obvious that tetrahedral or triangular elements are advantageous because of elemental vertex angles that allow locally angled crack paths. Other authors claim that the shape of the element is critical to realizing an experimental crack path. Particularly, [4] claims that pinwheel-shaped elements possess an isoperimetric property that gives the mesh the ability to reproduce any curve as the element sizes decrease.

In fragmentation studies, the cohesive elements allow material elements to dislodge from the specimen. This changes the topology of the specimen, and the elements must become aware of the updated topology. A method of tracking and updating the topology is presented in [3]. Generally, fatigue studies will not need these types of updates because fragmenting is cause for total failure. 


\subsection{Computational Cost in Fatigue Acknowledgements Analyses}

It may be computationally expensive to run thousands of cycles to obtain a completely decohered cohesive element. One can interpolate cyclic results to reduce the cost of their simulations. Also one could condition $\lambda$ to a higher value to account for multiple cycles in one "super-cycle".

One may want to apply pressure loadings to a specimen, but the convergence issues discussed here have not produced reliable results. Further literature research and/or investigation into altering the tangent stiffness matrix may yield more reliable methods to obtain convergence.

\section{Conclusions}

Fatigue studies require a mechanism to allow for void nucleation and crack initiation or propagation. Cohesive elements provide this mechanism with a permutable cohesive material law that accounts for both reloading and energy dissipation hysteresis. The use of cohesive elements provides the opportunity to use numerical studies to determine the effect upon fatigue of complex loading and geometric configurations not amenable to testing. Applications such as calculating the fatigue life of pressure vessel design are amenable to using cohesive elements since voids and cracks are delaminations of a material that precede the failure of a specimen. Future work will be to accurately predict delamination defects of components, and it is our hope that they can ultimately predict the necessity of replacing such components in the life of a power plant.

The authors gratefully acknowledge the Naval Nuclear Propulsion (NNP) Fellowship program for supporting this work performed at Bechtel Bettis, Inc. (BBI). Special thanks to Dr. Al DiNicola (BBI) for his ongoing support and insight into the methods and applications presented here, and to Dr. Ron Coffield (BBI) for supporting this work and future applications. Thanks to John Newman (BBI) for his gracious donation of numerical algorithms.

\section{References}

[1] Vinay K. Goyal, Eric R. Johnson, and Carlos G. Dávila, Irreversible constitutive law for modeling the delamination process using interfacial surface discontinuities, Composite Structures 65 (2004), no. $3-4,289-305$.

[2] Olivier T. Nguyen, E. A. Repetto, Michael Ortiz, and R. A. Radovitsky, $A$ cohesive model of fatigue crack growth, International Journal of Fracture 110 (2001), no. 4, 351-369.

[3] Anna Pandolfi and Michael Ortiz, An efficient adaptive procedure for three-dimensional fragmentation simulations, Engineering with Computers 18 (2002), 148-159.

[4] Katerina D. Papoulia, Stephen A. Vavasis, and Pritam Ganguly, Spatial convergence of crack nucleation using a cohesive finite element model on a pinwheel-based mesh, International Journal for Numerical Methods in Engineering 67 (2006), no. $1,1-16$.

[5] Gonzalo Ruiz, Anna Pandolfi, and Michael Ortiz, Three-dimensional cohesive modeling of dynamic mixed-mode fracture, 52 (2001), no. 1-2, 97-120. 